\title{
Deficits in Beam-Walking After Neonatal Motor Cortical Lesions Are Not Spared By Fetal Cortical Transplants In Rats
}

\author{
R.S. Swenson ${ }^{1}$, E.H. Danielsen ${ }^{1,2}$, B.S. Klausen ${ }^{1,2}$, E. Erlich ${ }^{1}$, J. Zimmer ${ }^{2}$ \\ and A.J. Castro ${ }^{1 *}$ \\ ${ }^{1}$ Department of Anatomy, Loyola University Stritch School of Medicine, Maywood, IL 60153, USA \\ and ${ }^{2}$ Institute of Neurobiology, Aarhus University, Aarhus, Denmark.
}

\begin{abstract}
Adult rats that sustained unilateral motor cortical lesions at birth demonstrated deficits in traversing an elevated narrow beam. These deficits, manifested by hindlimb slips off the edge of the beam, were not spared in animals that received fetal cortical transplants into the lesion cavity immediately after lesion placement.
\end{abstract}

\section{KEYWORDS}

sensorimotor cortex, transplant, motor, behavior

Fetal cortical tissue transplanted into sensorimotor neocortical lesion cavities of newborn rats survives, grows and develops connections with the host-brain /4-6/. These connections are qualitatively similar to those of normal sensorimotor cortex. Transplant efferents have been shown to arise from several thalamic subnuclei as well as from the basal forebrain, locus

\footnotetext{
Reprint address:

Anthony J. Castro, Ph.D.

Dept. of Anatomy

Loyola Univ. Stritch School of Medicine

2160 South First Ave.

Maywood, IL 60153, USA
}

VOL. 1, NO. 3, 4, 1989 coeruleus and dorsal raphe /4,5/. Transplant efferents project to the thalamus, cerebral cortex and as far caudally as the cervical spinal cord /4/. Additionally, fetal sensorimotor cortical grafts were found to respond to electrical stimulation of the contralateral forepaw /14/. In view of these anatomical and electrophysiological findings, the present study was designed to evaluate whether fetal cortical transplants are capable of ameliorating the effects of unilateral frontal cortical lesions on a common test of locomotor function, i.e., the ability to traverse a narrow beam.

Forty-one Long-Evans black-hooded rat pups were used. Under hypothermic anesthesia 22 animals sustained right frontal cortical lesions on postnatal day $0-1$. These lesions were located ju $k$ rostral to the coronal suture, $1-2 \mathrm{~mm}$ lateral to the midline and were made by aspiration of $1-2$ $\mathrm{mm}^{2}$ of cortical tissue down to the subcortical white matter. Thirteen of these rats received transplants of fetal frontal neocortex into the lesion cavity (NeoRFC Les + TP) immediately after lesion placement. This procedure which has been described previously /4,5/ involved the removal of embryonic day 14-15 fetuses from sodium pentobarbital anesthetized $(50 \mathrm{mg} / \mathrm{kg}$ ) dams. Fetal presumptive sensorimotor cortex was dissected in sterile Ringer's solution and the dissected tissue was gently aspirated into a glass pipette affixed to a Hamilton syringe. The graft was then slowly injected into the cortical aspiration cavity and held in place by a flap of the cartilaginous skull. After transplantation surgery, 
the donor rats were killed by intracardiac injection of sodium pentobarbital.

The remaining nine animals with frontal cortical lesions did not receive transplants (NeoRFC Lesion). An additional group of ten unoperated rats (Control) and a group of nine animals that were only hypothermically anesthetized at birth (Hypothermic) were also used.

All animals were subjected to the beam walking test at nine weeks of age. This test has been commonly applied to the evaluation of motor system lesions and has been reported to accurately reflect the laterality of large cortical lesions $/ 12 /$. The testing apparatus consisted of a wooden beam $5 \mathrm{ft}(150 \mathrm{~cm})$ long and 0.75 in $(1.9$ $\mathrm{cm})$ wide. The beam was covered with white plastic tape and elevated 15 in $(38 \mathrm{~cm})$ above a table. A partially enclosed dark box was placed at one end. Before each trial, the rat's hind paws were painted with a nontoxic water-based paint. The rat was then placed on the beam at a starting line on the side opposite the dark box. During three days of practice sessions, animals quickly learned to cross the beam and enter the box. The number of forelimb slips were counted by the examiner as the animals were performing the task. Hindlimb mis-steps could be observed as stripes of paint along the side of the beam. Results were collected over a distance of $3 \mathrm{ft}$ ( 90 $\mathrm{cm}$ ) on the center of the beam. After each trial, the paint was wiped off the beam. After the practice sessions, the performance of each rat was measured from five trials/day during a nine day period. All testing was done by the same investigator from 9-12 AM in a quiet, isolated room.

After testing, all animals were overdosed with sodium pentobarbital and perfused transcardially with $250 \mathrm{ml}$ of a $0.8 \%$ sucrose $-0.9 \%$ saline solution, followed by $650-700 \mathrm{ml}$ of a solution containing $10 \%$ formalin, $3.6 \%$ sucrose and $2.5 \%$ $\mathrm{MgSO}_{4}$. The brains were removed and frozen until sectioned frontally at $30 \mu \mathrm{m}$ on a cryostat. Every fourth section was stained with toluidine blue, and adjacent sections were stained for acetylcholinesterase.

Statistical analysis was done using the Tukey-
Kramer multiple comparison test with $\mathrm{p}<0.05$ accepted as significant.

\section{EFFECT OF NEONATAL RIGHT FRONTAL CORTICAL LESIONS}

Figure 1 presents the results of testing at nine weeks of age. Untreated Control and Hypothermic control animals were adept at negotiating the narrow beam with few errors in foot placement. They rarely made mistakes causing slips with their forepaws although left and right hindlimb slips were occasionally observed.

Animals with neonatal cortical lesions (NeoRFC Lesion) had significantly worse hindlimb performance, as indicated by the higher number of hindlimb slips on the beam, than either the Control or the Hypothermic animals. Notably, both left and right hindlimb slips of the NeoRFC Lesion animals were increased when compared to the respective limbs of Control and Hypothermic animals. The bilateral effect of the neonatal surgery is clearly different from the contralateral effect reported for hemidecortication in adult animals /12/. However within the bilateral deficits observed in our animals, the contralateral hindlimb showed more slips than the ipsilateral hindlimb $(\mathrm{p}<0.05)$. Our results also differ from observations that neonatal hemidecortication does not significantly increase the number of hindlimb foot-faults on a beam walking test, i.e., the neonatal animals were spared the effects of adult lesion /12/. However, this previous study used a broader and therefore somewhat less challenging beam.

The increased slipping of both hindlimbs in the NeoRFC Lesion group indicates a diminished ability to accurately place both hindlimbs following unilateral motor cortex lesions, suggesting a rather general, lesion-induced reorganization of motor systems rather than a specific involvement of contralateral limb function. Indeed, after unilateral ablation of one motor cortex, previous work has shown that the intact cerebral hemisphere gives rise to an anomalous 


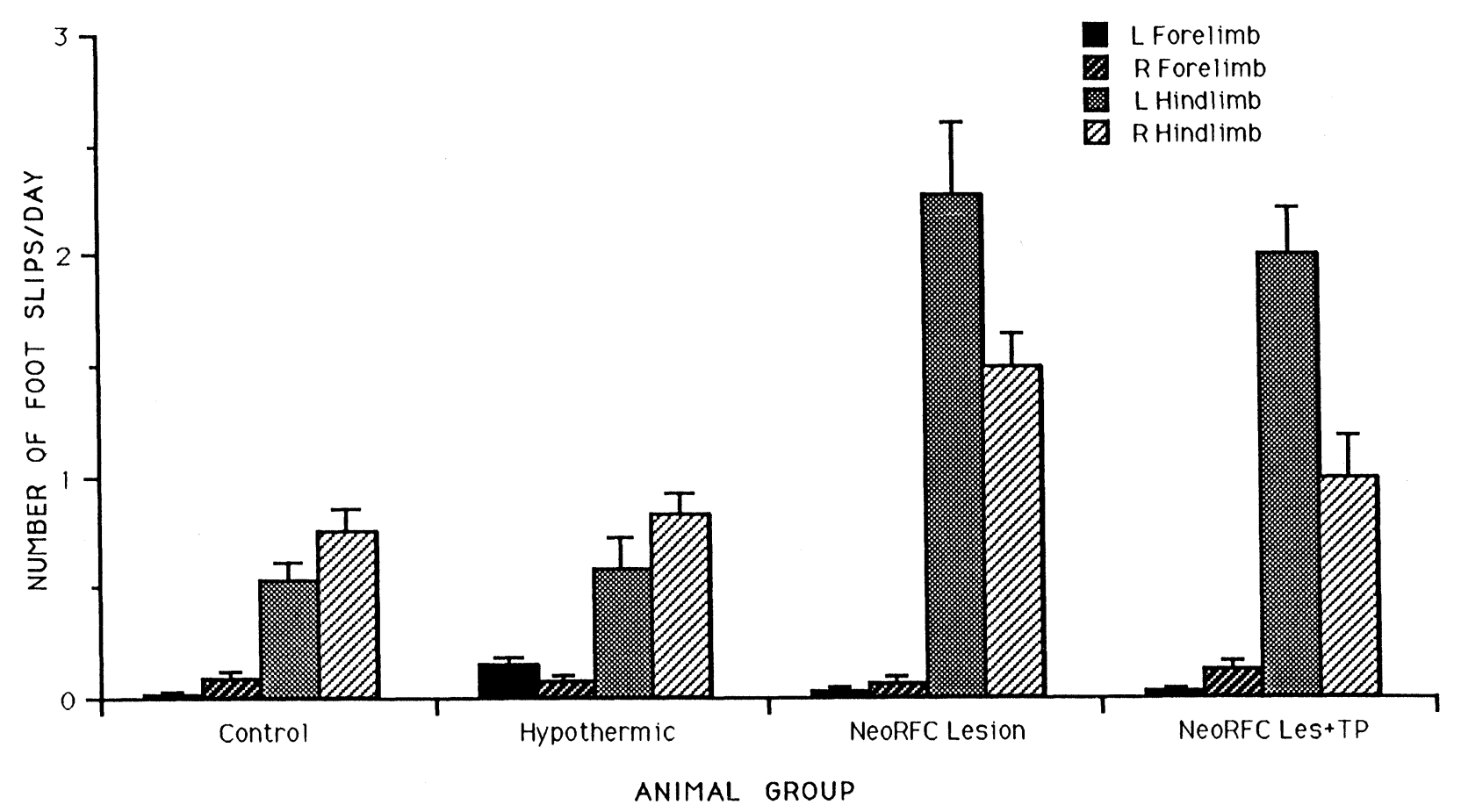

Fig. 1: Performance on beam walking test for normal Control, Hypothermic anesthetized, neonatal right frontal cortical lesion (NeoRFC Lesion) animals and lesion animals that received fetal cortical transplants (NeoRFC Les + TP). Values are expressed as the mean \pm 1 standard deviation. ipsilateral as well as the normal contralateral corticospinal tract $/ 1,9 /$. This abnormal, bilateral corticospinal projection corresponded to bilateral limb movements evoked by intracortical microstimulation of the spared cortex /10,11/. This bilateral effect differs from the pattern seen in normal rats where intracortical microstimulation primarily evokes only contralateral limb movements /13/.

In addition to corticospinal tract remodelling after neonatal cortical lesions, several reports demonstrate the remodelling of cortico-brainstem efferents after unilateral neonatal cortical lesions $/ 3 /$. This plasticity is commonly observed as a bilateral distribution of projections that normally are primarily unilateral. These bilateral cortico-brainstem and corticospinal projections may therefore enable the spared cerebral hemisphere to assume a bilateral function. Alternatively, these bilateral cortical connections may actually interfere with normal locomotor func- tions as suggested by the hindlimb data in our study.

Despite the poor performance of our animals' hindlimbs on the beam walking test, forelimb slippage after neonatal motor cortical lesions was not significantly different from controls. This finding correlates with the spared functions of reaching and grasping which have been observed following similar lesions of neonatal rat cortex $/ 2,8,12 /$.

\section{FETAL CORTICAL TRANSPLANTS}

Of the 13 animals receiving grafts of fetal frontal cortex, 12 were found to have transplants. The histological appearance of such grafts has been previously described $/ 3,4,5 /$. Five animals demonstrated particularly large transplants that extended into the striatum, and one animal showed a small transplant poorly connected to 
the surrounding cortex. The remainder of the grafts were well integrated into the host cortex and typically filled the lesion cavity extending from the c.rtical surface down to the subcortical white matter.

The poor hindlimb performance in animals sustaining cortical lesions as neonates was not improved by transplantation of fetal cortical tissue into the lesion cavity (Fig. 1). Similar to animals only receiving lesions, the transplant group of animals showed more contralateral than ipsilateral hindlimb slips $(p<0.01)$. Although the right hindlimb was somewhat better on average than the right hindlimb of animals without transplants, this difference was not significant. Further analysis of the data from this group showed that transplant size was not a factor in limb function. The six animals with the largest grafts had an average of $2.22 \pm 0.39$ and $1.22 \pm 0.29$ slips/test day for the left and right hindlimb, respectively. The corresponding values for the six animals with the smallest transplants were 1.76 \pm 0.16 and $0.76 \pm 0.24 \mathrm{slips} /$ day. Although the group with large transplants had more slips on the average, this was not a significant difference ( $p>0.2$ ).

Previous reports from our laboratory have shown that fetal neocortical transplants survive and grow when they are placed in frontal cortical lesion cavities. Additionally, these transplants develop connections with the host brain which are characteristic of motor cortex /4-6/. These transplants also have been shown to receive physiologically active somatosensory input /14/. However, utilizing tests of an animal's ability to traverse a narrow beam, the present study identified no significant sparing of function. This lack of functional sparing is compatible with reports by Dunnett et al /7/ who found no long-term recovery from learning deficits produced by prefrontal cortex lesions. However, contrary to their report, we did not find greater impairments in our animals with fetal cortical grafts than with lesion alone.

\section{ACKNOWLEDGEMENTS}

Supported by NIH grant NS 13230 and by funds from the Danish MRC. We are grateful to Ms. Pamela Shaw and Mr. Thomas Hogan for technical assistance and to Dr. E.J. Neafsey for reading the manuscript.

\section{REFERENCES}

1. Castro AJ. Ipsilateral corticospinal projections after large lesions of the cerebral hemisphere in neonatal rats. Exp Neurol 1975; 46: 1-8.

2. Castro AJ. Limb preference after lesions of the cerebral hemisphere in adult and neonatal rats. Physiol Behav 1977; 18: 605-608.

3. Castro AJ. Plasticity in the motor system. In: Kolb BE, Tees RC (eds.), The Cerebral Cortex of the Rat. MIT Press, 1989 (In press).

4. Castro AJ, Tфnder N, Sunde N, Zimmer J. Fetal cortical transplants in the cerebral hemisphere of newborn rats: a retrograde fluorescent analysis of connections. Exp Brain Res 1987; 66: 533-542.

5. Castro AJ, T $\phi$ nder N, Sunde N, Zimmer J. Fetal cortical transplants grated into the cerebral cortex of newborn rats. Analysis of efferents from the basal forebrain, locus coeruleus and midline raphe. Exp Brain Res 1988; 69: 613-622.

6. Castro AJ, Zimmer J, Sunde NA, Bold EL. Transplantation of fetal cortex to the brain of newborn rats. A retrograde fluorescent analysis of callosal and thalamic projections from transplant to host. Neurosci Lett 1985; 60: 283-288.

7. Dunnett SB, Ryan CN, Levin PD, Reynolds $M$, Bunch ST. Functional consequences of embryonic neocortex transplanted to rats with prefrontal cortex lesions. Behavioral Neurosci 1987; 101: 489-503.

8. Gentile AM, Green S, Nieburgs A, Schmelzer W, Stein DG. Disruption and recovery of locomotor and manipulatory behavior following cortical lesions in rats. Behavioral Biol 1978; 22: 417-455.

9. Hicks SP, D'Amato CJ. Motor-sensory and visual behavior after hemispherectomy in newborn and mature rats. Exp Neurol 1970; 29: 416-438.

10. Kartje-Tillotson G, Neafsey EJ, Castro AJ. Electrophysiological analysis of motor cortical plasticity after cortical lesions in newborn rats. Brain Res 1985; 332: 103-111.

11. Kartje-Tillotson G, O'Donoghue DL, Dauzvardis MF, Castro AJ. Pyramidotomy abolishes the abnormal movements evoked by intracortical microstimu- 
lation in adult rats that sustained neonatal cortical lesions. Brain Res 1987; 415: 172-177.

12. Kolb B, Tomie J. Recovery from early cortical damage in rats. IV. Effects of hemidecortication at 1,5 , or 10 days of age on cerebral anatomy and behavior. Behav Brain Res 1988; 28: 259-274.

13. Neafsey EJ, Bold EL, Haas G, Hurley-Geis KM, Quirk G, Sievert CF, Terreberry RR. The organiza- tion of the rat motor cortex: a microstimulation study. Brain Res Rev 1986; 11: 77-96.

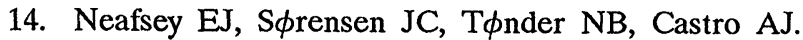
Fetal cortical transplants into neonatal rats respond to thalamic and peripheral stimulation in the adult. An electrophysiological study of single-unit activity. Brain Res 1989; 493: 33-40. 

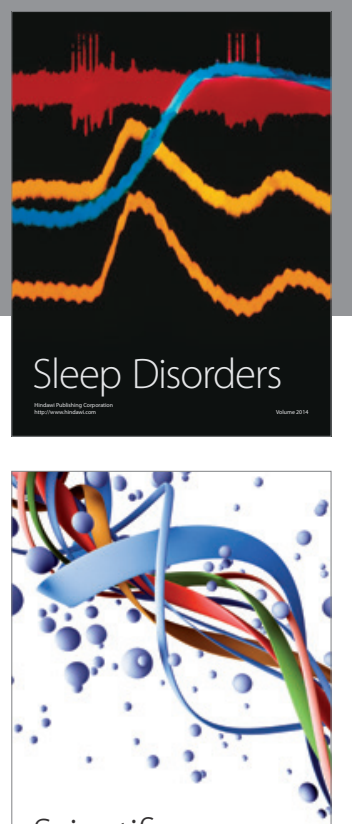

Scientifica
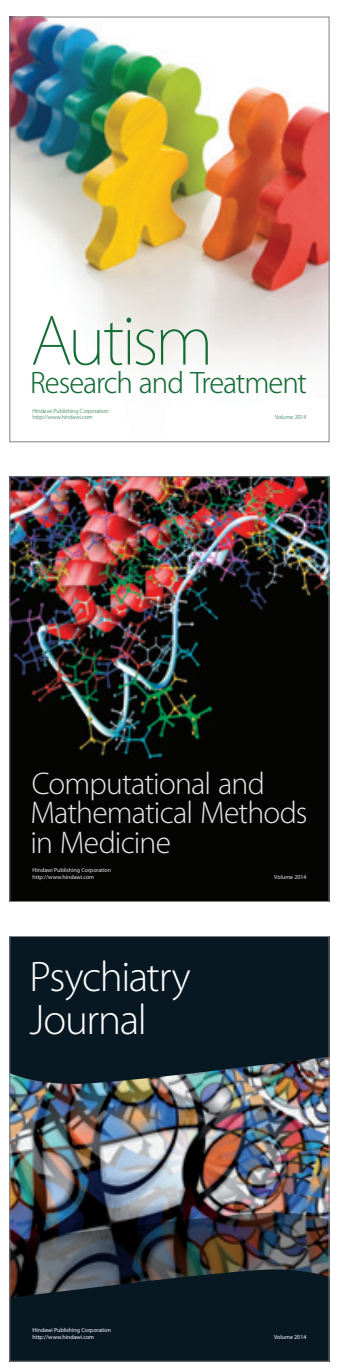
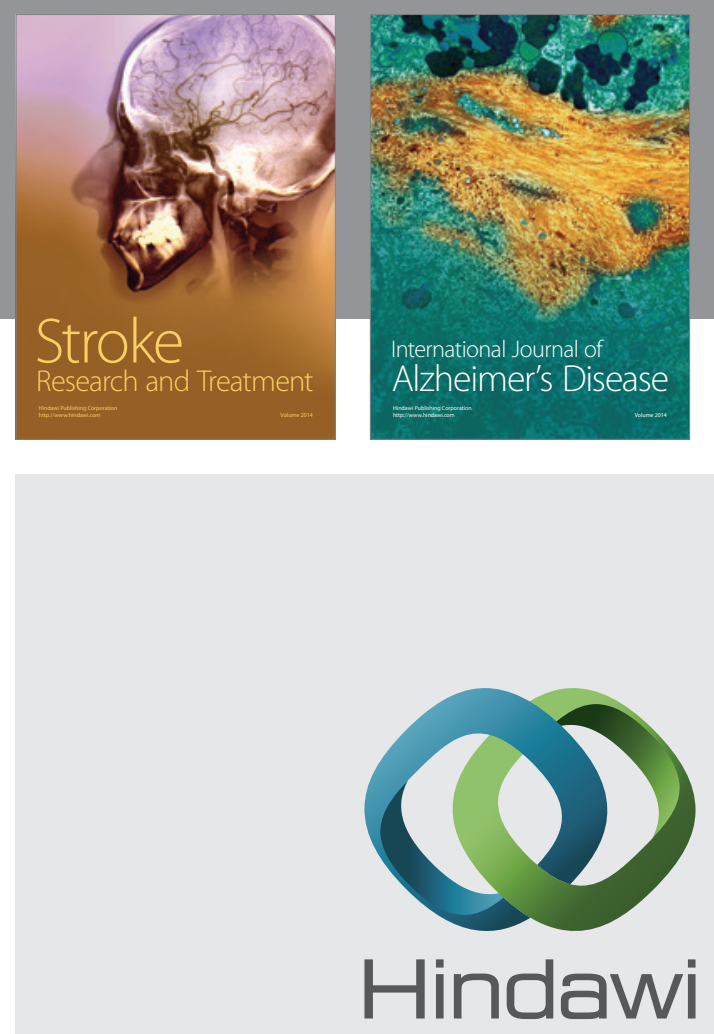

Submit your manuscripts at

http://www.hindawi.com
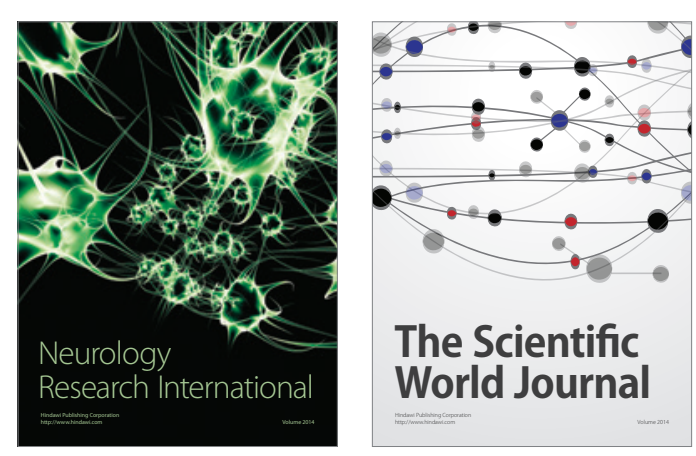

The Scientific World Journal

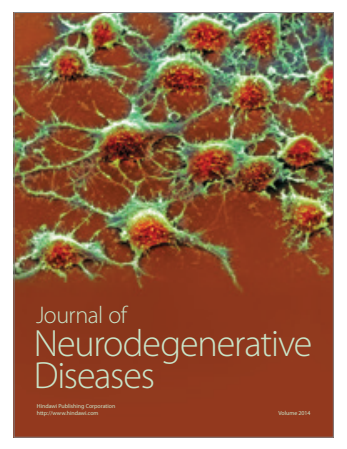

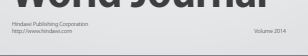

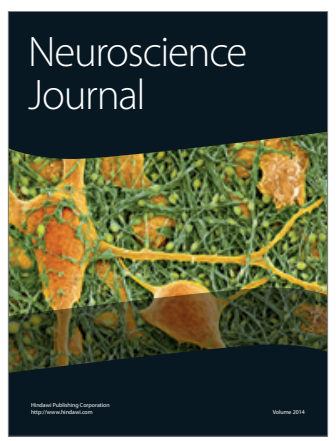

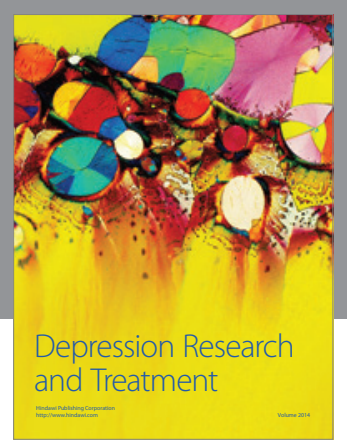
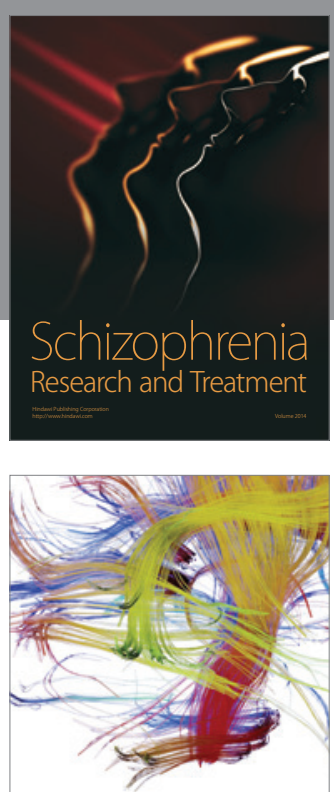

Brain Science

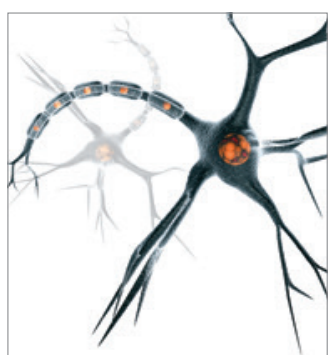

Neural Plasticity
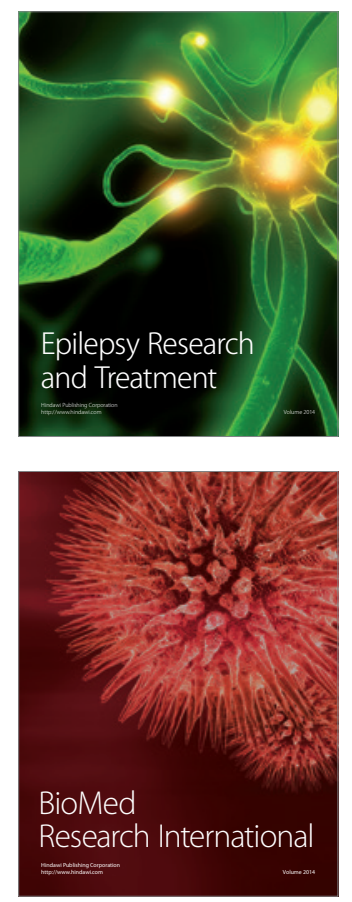

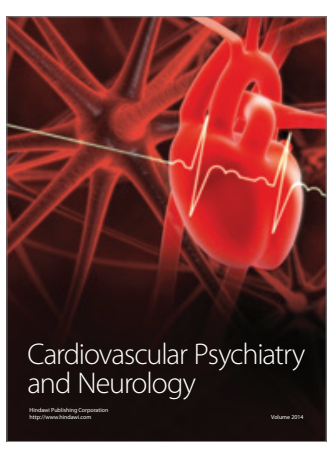

Parkinson's

Disease
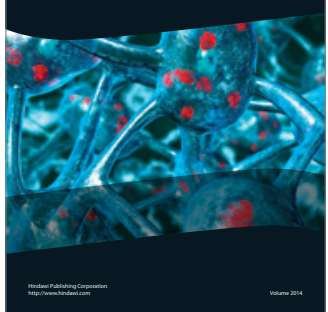